\title{
Parental Acceptance-Rejection, Childhood Trauma, Emotion Regulation, and Psychological Adjustment as the Risk Factors of Psychopathic Tendencies in Adolescents of Pakistan
}

\author{
Saba Walayat', Mussaffa Butt ${ }^{2}$
}

\begin{abstract}
The present study aimed to explore the psychopathic tendencies in relation to childhood trauma, psychological adjustment, emotion regulation and parental patterns of acceptance-rejection towards their adolescents and the influence of demographic variables in socio-cultural context of Pakistan. Translated versions of Parental Acceptance-Rejection Questionnaire; Child PARQ, Childhood Trauma Questionnaire, Personality Assessment Questionnaire, Emotion Regulation Questionnaire, Youth Psychopathic Trait Inventory and bio-data form containing information regarding different demographic variables were used in order to collect the data from the participants. The sample consisted of 600 adolescents ( 281 males; 319 females) with age ranged between 13 and 17 were selected with convenient random sampling from different private and public schools of the three major cities of Punjab; Lahore, Gujrat and Sialkot. The results indicated psychological maladjustment as the most significant predictor of psychopathic tendencies. However, emotion regulation appeared to be as the most significant predictor of psychopathic tendencies in male adolescents and parental acceptance-rejection (mother) in female adolescents. The results further indicated that age, father's education and profession, mother's education and monthly income are significant determinants of psychopathic tendencies in participants of the study.
\end{abstract}

Keywords: Childhood trauma, emotion regulation, parental acceptance-rejection, psychopathic tendencies. JEL Codes: M3, M31.

Available Online: 10-06-2017

DOI: http://dx.doi.org/10.18533/ijbsr.v7i5.1048

This is an open access article under Creative Commons Attribution 4.0 License, 2017.

\footnotetext{
${ }^{1}$ Government College University, Lahore, Pakistan. Email: sabawalayatkhan@gmail.com

${ }^{2}$ Government College University, Lahore, Pakistan. Email: mussaffa@gcu.edu.pk
} 


\section{INTRODUCTION}

Psychopathy has captured the attention of so many scientists due to the relative rarity of the disorder and the severity of its impact on society. Psychopathy and psychopathic tendencies in relation to other variables have been an area of immense importance. Studies have consistently indicated a noteworthy connection of psychopathic tendencies with childhood trauma, interpersonal acceptance-rejection, emotional regulation and psychological adjustment. Culture also plays a substantial role in development of psychopathic tendencies. Therefore, the present study is aimed to explore relationship of these variables with psychopathic tendencies in socio-cultural context of Pakistan.

Psychopathy as a personality disorder associated with the presence of superficial charm, insincerity and untruthfulness, pathologic egocentricity, and lack of shame or remorse (Cleckley, 1941). Perhaps the psychopathic personality has captured the attention of so many scientists due to the relative rarity of the disorder and the severity of its impact on society. Base rates for psychopathy are generally very low, despite higher rates within the prison population (15-25\%) (Hare, 2003). Although small, this group of individuals is perceived to be responsible for a disproportionally large amount of crime and resulting financial loss to society (Hare, McPherson, \& Forth, 1988; Hart, Kropp, \& Hare, 1988; Moffitt, 1993). Psychopathic individuals are usually at an increased risk of reoffending and their attempts are usually more often, quick and violent as compare to non-psychopathic offenders (Salekin, Rogers, \& Sewell, 1996). Furthermore, research generally agrees on the idea that men show higher levels of psychopathy than women (Cale \& Lilienfield, 2002a; Verona \& Vitale, 2006; Vitale \& Newman, 2001).

Therefore, it has been appeared to be as the subject of increased importance for researchers to examine psychopathy among adolescents, although typically studied in adult criminal offenders (Frick, 1995; Lynam, 1996). However, we are still not able to have that collateral evidence that what we are assessing in children and adolescents is certainly a psychopathy, a personality disorder which will expected enough to remain quite stable within individuals who are moving into their adulthood (Hart, Watt, \& Vincent, 2002).

Up until now, there is little convincing longitudinal evidence that individuals who show psychopathic tendencies in childhood will remain highly psychopathic, while moving through the developmental transitions into adolescence and then into adulthood. The results of numerous small studies propose that measures of juvenile psychopathy demonstrate moderate to high rank-order stability from late childhood to early adolescence, with a context where the same informant (e.g., a parent) repeatedly completes the measures (Andershed, 2010).

Some of the important researches and the past theoretical background provided us with the link of psychopathy with childhood trauma, Interpersonal acceptance/rejection theory and emotional regulation.

Studies proposed an important connection between childhood trauma (resulting from child abuse) and psychopathic tendencies. For example, Weiler and Widom (1996), in elaborating the probable mechanisms by which psychopathy mediated the abuse-violence bondage in their sample, proposed that as a result of early abuse, a child has a tendency to become 'desensitized' to painful or anxiety provoking experiences of future and therefore as a result of this desensitization, he might become less physiologically and emotionally responsive to the needs of others, lack empathy and to be callous, and also having a deficiency of situationally appropriate remorse or guilt. On the other hand, Cohen, Mannarino, and Deblinger (2006) have found that trauma during early childhood may have even greater consequences for developmental trajectories than traumas that occur in later adolescence.

Some researchers have proposed that abuse is the result of reduced capacity of an individual to respond with empathy. Studies support a notable connection between childhood trauma and psychopathic tendencies. Likewise, Coupland and Moretti (2010) showed that maternal physical abuse was considerably linked with psychopathy in males but not females. Maternal physical abuse was also linked 
with Post Traumatic Stress disorder, hyper-arousal for males only and of PTSS re-experiencing for both males and females. Paternal physical abuse was not considerably linked to psychopathy or PTSS for males or females.

On the other hand, Individuals with psychopathic tendencies may have very diverse patterns of responses to emotional stimuli, both of which could contribute to their problems in conscience development. Therefore, theories of moral development propose that patterns of emotional underarousal and over-arousal may both get in the way with the normative conscience development (Frick \& Morris, 2004; Hoffman, 1982; Young, Fox, \& Zahn-Waxler, 1999). Available research has quoted this idea that individuals with psychopathic tendencies appear to exhibit differences in their emotional and cognitive processing. Number of studies has showed that psychopathy is an acquired state of emotional disturbance, so the researchers Donahue, McClure and Simon (2014) indicated that the difficulties of emotion regulation were negatively connected with Fearless Dominance psychopathic traits, but on the other hand, positively linked with global psychopathic traits and Self-Centered Impulsivity.

However, Psychopathic tendencies can be the result of maladjustment which in fact expected to be linked up with perceived rejection by parents. Parental rejection has been explored to be constantly connected with psychological adjustment in number of ways. In both clinical and non-clinical depression appeared to be an important outcome as the result of maladjustment within major ethnic groups in the United States, including African Americans (Crook, Raskin, \& Eliot, 1981), Asian Americans (Greenberger \& Chen, 1996), European Americans (Belsky \& Pensky, 1988; Jacobson, Fasman, \& DiMascio, 1975; Whitbeck, Conger, \& Kao, 1993; Whitbeck, Hoyt, Miller, \& Kao, 1992), and Hispanic Americans (Dumka, Roosa, \& Jackson, 1997). Parental rejection also seems to be a major predictor of almost all types of behavior problems, including conduct disorders, externalizing behavior, and delinquency. Cross-cultural evidence that support this conclusion come from Bahrain (Al-Falaij, 1991), China (Chen, Rubin, \& Li, 1997), Croatia (Ajdukovic, 1990), Egypt (Salama, 1984), England (Farrington \& Hawkins, 1991; Maughan, Pickles, \& Quinton, 1995), India (Saxena, 1992), and Norway (Pedersen, 1994).

Baker and Hoerger (2012) found that both parental over control and rejection were appeared to be related to general deficits in self-regulation along with psychopathology and adjustment difficulties. Parental rejection was linked with internalizing clinical presentations such as depression, anxiety, and somatization, whereas over control was more considerably linked with psychoticism and increased hypomanic activation. Further, it had also been found that self-regulation partially mediated the relationships between parental child-rearing strategies, socio-emotional adjustment and psychopathology.

Some of the Meta-analyses and literature reviews conducted in this direction also contributed in developing our understanding regarding the relation between parenting and psychopathy. Smith (2011) conducted the literature review which aimed to investigate the evidence for a link among insecure attachment and the development of psychopathy. The Rorschach studies specifically propose that an inability to make attachments may be a central aspect of psychopathy. In the same way, Shahid et al (2009) analyzed from their study that paternal rejection is positively correlated with psychoticism but negatively correlated with extraversion. The result revealed that there was no significant correlation between paternal rejection and neuroticism.

There is a distressing increase in the incidence of mental illness due to a result of continuous wave of violence, political chaos and recurrent changes in the social fabric across many countries in the world (World Health Organization, 2001; 2005). A wide range of psychiatric disorders have been reported, as a result of such changes, like depression, substance and alcohol misuse, bipolar disorder, post-traumatic stress disorder, schizophrenia \& psychopathic tendencies (Gadit,2007). Suicide rate has been tremendously increased every year in both of the developed and developing countries as a product of mental health problems (Gadit, 2007). 
Mental health problems in a developing country like Pakistan, have reached an appalling level in the last few decades (David \& Malik, 2000; Gadit, 2005) connected to both the existing violence in Pakistani society (Gadit \& Vahidy, 1999; Khalily, Fooley, Hussain \& Bano, 2011) and disturbance in its social structure (Gadit \& Vahidy, 1999). Many researchers acknowledged that as the whole psychological outcome of the communities will be that a matter of resilience rather than psychopathology (Galea, Ahern, Resnick, Kilpatrick, Bucuvalas \& Gold, 2002). However, a greater degree of exposure to traumatic events is known to be linked with the greater occurrence of severe problems of mental health (Galea, Vlahov, Resnick, Ahern, Susser \& Gold, 2003). This persisting wave of violence and threat to life has had a disrupted effect to the psychological health of many people in general (Marzuk, 1996) and predominantly in Pakistani society (Khalily et al., 2011).

The above mentioned empirical studies showed that it is extremely important to detect psychopathic tendencies as early as possible. Adolescents are highly vulnerable for the development of different psychological, emotional, and behavioral problems (Caspi, Taylor, Moffitt, \& Plomin 2000; Rowling, 2006). Adolescence is the most crucial period of human development marked by immense biological, emotional, social and cognitive changes. Societies need better understanding regarding all aspects of adolescence including psychopathic tendencies and its relation with aspects like childhood trauma, psychological adjustment and their perception of adolescents about parental behaviors. These problems might be demonstrated in terms of social, academic, emotional, and psychological difficulties. These problems may result into serious negative consequences (Saluja, lachan, Scheidt, Overpech, Sun \& Gidd, 2004; Slemming, Sorensen, Thomsen, Obel, Henriksen \& Linnet, 2010). Adolescents are at edge of developing complexities influenced by emotional changings so it is also considered to be important to study the relation of childhood trauma, parental acceptance-rejection, psychological adjustment and emotional regulation with psychopathic tendencies. Moreover, the interrelationship between all these variables have also been supported by research conducted in different cultures; like the evidence of significant relation between childhood trauma and emotion regulation (Shipman, Schneider, Fitzgerald, Sims, Swisher, \& Edwards, 2007) and also between perceived rejection by parents and psychological maladjustment (Rohner, 1975).

Furthermore, culture plays a significant role in the development of adolescents. Research has indicated that psychopathy greatly influenced by norms and values within a given perceptual background of a culture. In socio-cultural context of Pakistan, it is far more important to take the holistic picture of psychopathic tendencies because as a nation we are suffering immensely from different type of extremism which may take to violence and bloodshed. The targeted population in such activities are youth (majorly adolescents) for suicide bombing and sectarian violence. Literature showed that individuals with psychopathic tendencies are usually at an increased risk of reoffending (Salekin, Rogers, \& Sewell, 1996).

It's important to explore the possible risk factors beneath the cover which can potentially trigger the development of psychopathic tendencies. In socio-cultural context of Pakistan, there is serious dearth of literature on this extremely important issue. The present study is one such effort which aims to explore whether psychopathic tendencies do exist in our culture or not, are they equally evident in both genders of adolescents and that do they also significantly related with other variables of the research like childhood trauma, parental acceptance-rejection, psychological adjustment and emotion regulation.

\subsection{OBJECTIVES}

1. To investigate the relationship between parental acceptance/rejection, childhood trauma, psychological adjustment and emotion regulation with psychopathic tendencies.

2. To investigate the relationship between parental acceptance/rejection, childhood trauma and emotion regulation with psychological adjustment.

3. To investigate the relationship between parental acceptance/rejection, childhood trauma and psychological adjustment with emotion regulation. 
4. To investigate the gender differences in experiences and presence of psychopathic traits, childhood trauma, parental acceptance/rejection, emotion regulation and psychopathic tendencies.

5. To determine the effects of demographic variables on parental acceptance/rejection, childhood trauma, psychological adjustment emotional regulation and psychopathic tendencies.

\subsection{HYPOTHESES}

1. Perceived parental rejection, childhood trauma, emotional dysregulation and psychological maladjustment will be the significant predictor of psychopathic tendencies.

2. Childhood trauma and perceived parental rejection will be significantly affect emotional dysregulation and psychological maladjustment.

3. Emotional dysregulation and psychological maladjustment would be significantly interrelated.

4. There would be significant gender differences in perceived parental acceptance-rejection, childhood trauma, emotional regulation, psychological adjustment and psychopathic tendencies.

5. There would be significant effect of demographic variables on perceived parental acceptancerejection, childhood trauma, emotional regulation, psychological adjustment and psychopathic tendencies.

\section{METHODOLOGY}

\subsection{SAMPLE}

The sample consisted of 600 adolescents. It had been ensured that the sample contained the equal representation of both genders. The participants selected for the research were school going adolescents of $9^{\text {th }}$ grade and $10^{\text {th }}$ grade. The age ranged from 13 to 17 years. The selection of the participants was based on convenient random sampling techniques. The data had been collected from private and public schools located in three different cities of Punjab; Lahore, Gujranwala and Sialkot, after taking permission from administration of concerned schools. The selected schools were located in different socio-economic statuses of the society to confirm the representation of all the major socioeconomic classes.

\subsection{MEASURES}

\subsubsection{BIO-DATA FORMS}

The bio-data forms were provided to the participants which contained different segments of personal information regarding age, education and the other aspects related to family like birth order, number of siblings, family income, father's and mother's education, father's and mother's profession and also about the possible conviction in family.

\subsubsection{PARENTAL ACCEPTANCE-REJECTION QUESTIONNAIRE CHILD VERSION (CHILD PARQ)}

This self-report questionnaire measures perceptions of parental treatment of the child in terms of four dimensions, a) parental warmth and affection, b) parental hostility and aggression, c) parental indifference and neglect, and c) parental undifferentiated rejection. Taken together, these four scales compose the total PARQ score used in this study. Scale contained 24 items and each subscale comprised of 6 items. Children responded to two versions of the PARQ (Rohner, 1990), one assessing children's perceptions of their father's (or primary male caregiver's) acceptance-rejection (Child PARQ, father version) and one assessing mother's (or primary female caregiver's) acceptance-rejection (Child PARQ, mother version). The questionnaire is keyed in the direction of perceived rejection. The higher the score, the more rejection children tend to experience. The lower the score, the more acceptance children tend 
to experience. For the present study, translated version of the original questionnaires has been used, developed by Malik (2011).

\subsubsection{PERSONALITY ASSESSMENT QUESTIONNAIRE (PAQ)}

This self-report questionnaire (Rohner, 1990) evaluates the way in which children perceive their own personality and behavioral dispositions. The following scales constitute the child PAQ: a) hostility/aggression, b) dependence, c) negative self-esteem, d) negative self-adequacy, e) emotional unresponsiveness, $f$ ) emotional instability, and $g$ ) negative world views. The PAQ is keyed in such a way so that the higher the score, the higher the level of self-reported psychological maladjustment. For the present study, translated version of the original questionnaire is used, developed by Naz \& Kauser (2011).

\subsubsection{CHILDHOOD TRAUMA QUESTIONNAIRE (CTQ)}

The original CTQ was developed from a 70-item retrospective questionnaire for which participants were required to rate the frequency (o- never true to 5-very often true) of abuse and neglect events that took place when they "were growing up" (Bernstein, Fink, Handelsman, Foote, \& Lovejoy, 1994). In further studies, the length of the scale was reduced to 28 items based on exploratory and confirmatory factor analyses (Bernstein, Stein, Newcomb, Walker, \& Pogge, 2003). The short version of the CTQ assesses emotional abuse, physical abuse, sexual abuse, emotional neglect, and physical neglect. The translated version of the original CTQ has been used for the present study developed by Butt, Khan, Malik and Sharif (2013).

\subsubsection{YOUTH PSYCHOPATHY TRAITS INVENTORY (YPI)}

The YPI is a 50-item youth self-report questionnaire designed to measure traits of psychopathy (Andershed, Stattin \& Levander 2002). The YPI uses a 4-point Likert type response scale ranging from $1=$ does not apply at all to $4=$ applies very well and measures each psychopathic trait with five self-report items making up 10 internally consistent subscales. Participants can score between 50 and 200, where the high scores indicated the high presence of psychopathic traits and the low scores indicated the presence of less psychopathic traits. The subscales of the YPI have been shown to form a theoretically meaningful and useful three-factor structure consisting of (a) the Grandiose/Manipulative factor, including the subscales Dishonest Charm, Grandiosity, Lying, and Manipulation; (b) the Callous/Unemotional factor, including the subscales Callousness, Unemotionality, and Remorselessness; and (c) the Impulsive/Irresponsible factor, including the subscales Impulsiveness, Thrill-Seeking, and Irresponsibility. The translated version of the original YPI has been used for the present study developed by Butt, Saeed, Walayat and Malik (2013).

\subsubsection{EMOTION REGULATION QUESTIONNAIRE (ERQ)}

The ERQ is a 10-item self-report measure of an individual's tendency to use reappraisal and expressive suppression to regulate emotion. Each item consists of a seven-point Likert scale (1 strongly disagree; 7 strongly agree). The participants can score between 10 and 70 where the high score on the scale indicated high emotion regulation while the low score mentioned the low emotion regulation. The ERQ consists of reappraisal (six items) and expressive suppression (four items) subscales, with subscales scored as the mean of the items. For the present study, translation of the original measure has been used, developed by Butt, Malik and Kaleem (2012).

\subsection{PROCEDURE}

It was considered to be important to take the prior permission from the school authorities while explaining them the objectives of the study because of to its critical nature. With the help of class room teachers, the participating children were approached and were provided with informed consent before administering the questionnaire consisted of youth psychopathic trait inventory, PARQ for mother and 
father, childhood trauma questionnaire, personality assessment questionnaire, emotion regulation questionnaire along with a bio-data form. They were instructed to feel free if they had any queries and carefully complete the questionnaires.

\section{RESULTS}

Data collected for the present study were analyzed by using Statistics IBM SPSS 20 (International Business Machines Corporation; Statistical Package for Social Sciences. 20.0.0) Appropriate statistical analyses were carried out to test the hypotheses in relation with the objectives of the study.

The statistical procedures like correlation, regression analysis and between-within ANOVA were employed to determine the relationship of psychopathic traits with different variables under consideration and differences across groups of adolescents on the basis of gender. Adolescents were grouped into male $(n=281, M=43.79, S D=11.95)$ and female $(n=319, M=46.5, S D=12.04)$.

The correlation of psychopathic traits was highly significant with childhood trauma $(r=.17, p<.01)$, subscales of emotion regulation; cognitive reappraisal $(r=.13, p<.01)$ and expressive suppression $(r=.19$, $p<.01)$, parental acceptance-rejection (mother) $(r=.17, p<.01)$ and psychological adjustment $(r=.22, p<.01)$, while correlation was also significant with parental acceptance-rejection (father) $(r=.09, p<.05)$.

The correlation analysis was separately conducted for both groups which showed that in male adolescents' psychopathic traits had highly significant correlation with subscales of emotion regulation; cognitive reappraisal $(r=.26, p<.01)$, expressive suppression $(r=.39, p<.01)$ and psychological adjustment $(r=.24, p<.01)$ while had significant correlation with childhood trauma $(r=.19, p<.05)$ and parental acceptance-rejection (mother) $(r=.10, p<.05)$. However, the correlation between psychopathic traits and parental acceptance-rejection (father) was not significant ( $r=-.01, p=n . s)$. In case of female adolescents, psychopathic traits had highly significant correlation with parental acceptance-rejection (father) $(r=.15$, $p<.01)$, parental acceptance-rejection (mother) $(r=.23, p<.01)$ and psychological adjustment $(r=.22, p<.01)$. However, non-significant correlation had been reported with childhood trauma $(r=.10, p=n . s)$ and subscales of emotion regulation ( $r=.03, p=n . s)$.

Stepwise regression analysis indicated psychological maladjustment as the most significant predictor of psychopathic traits, $\beta=.17$, whereas all other demographic variables like education, SES, family type and family size were excluded.

Table 1: Predictors of youth psychopathic traits

\begin{tabular}{llcl}
\hline Model & B & SE & $\beta$ \\
\hline Step 1 & & & \\
Psychological adjustment & .24 & .06 & .17 \\
Cognitive reappraisal & .33 & .11 & .13 \\
Expressive suppression & .43 & .14 & .13 \\
Childhood trauma & .20 & .06 & .15 \\
Step 2 & & & \\
Psychological adjustment & .23 & .06 & .16 \\
Cognitive reappraisal & .33 & .11 & .13 \\
expressive suppression & .44 & .13 & .14 \\
Childhood trauma & .20 & .06 & .15 \\
Step 3 & & & \\
Psychological adjustment & .26 & .06 & .18 \\
Cognitive reappraisal & .32 & .11 & .13 \\
Expressive suppression & .45 & .13 & .14 \\
childhood trauma & .22 & .06 & .17 \\
\hline
\end{tabular}

Step 1: $\mathrm{R} 2=.11, \mathrm{p}<.01$. Step $2: \mathrm{R} 2=.11, \mathrm{p}<.01$. Step $3: \mathrm{R} 2=.11, \mathrm{p}<.01$. 
Apart from psychological maladjustment, other significant predictors were cognitive reappraisal $(\beta=.13)$ and expressive suppression $(\beta=.13)$ of emotion regulation and childhood trauma $(\beta=.15)$. In case of subscales of psychological adjustment questionnaire, aggression and emotional stability were the significant predictors of psychopathic traits in adolescents whereas in childhood trauma questionnaire, emotional abuse was the only significant predictor of youth psychopathic traits.

Table 2: Subscales of psychological adjustment questionnaire as predictors of youth psychopathic traits

\begin{tabular}{llll}
\hline Model & B & SE & $\beta$ \\
\hline Step 1 & & & \\
Aggression & .90 & .24 & .17 \\
Emotional stability & .54 & .26 & .10 \\
Step 2 & & & \\
Aggression & .90 & .24 & .17 \\
Emotional stability & .54 & .25 & .10 \\
Step 3 & & & \\
Aggression & .90 & .24 & .17 \\
Emotional stability & .54 & .25 & .10 \\
Step 4 & & & \\
Aggression & .90 & .24 & .17 \\
Emotional stability & .54 & .25 & .10 \\
Step 5 & & & \\
Aggression & .95 & .23 & .18 \\
Emotional stability & .56 & .26 & .10 \\
Step 5 & & & .19 \\
Aggression & .97 & .23 & .11 \\
Emotional stability & .61 & .24 & \\
\hline Step & & & \\
\hline
\end{tabular}

Step 1: $\mathrm{R} 2=.06, \mathrm{p}<.01$. Step 2: $\mathrm{R} 2=.06, \mathrm{p}<.01$. Step 3: $\mathrm{R} 2=.06, \mathrm{p}<.01$.

Step 4: $R 2=.06, p<.01$. Step 5: $R 2=.06, p<.01$. Step 6: $R 2=.06, p<.01$.

Table 3: Subscales of childhood trauma questionnaire as predictors of youth psychopathic traits

\begin{tabular}{lccc}
\hline Model & B & SE & 8 \\
\hline Step 1 & & & .18 \\
Emotional abuse & .89 & .26 & .18 \\
Step 2 & & & \\
Emotional abuse & .88 & .26 & .19 \\
Step 3 & & .23 & .18 \\
Emotional abuse & .90 & .22 & .19 \\
Step 4 & & & \\
Emotional abuse & .88 & .19 & \\
Step 5 & & .91 &
\end{tabular}

Step 1: $R 2=.03, p<.01$. Step 2: $R 2=.03, p<.01$. Step 3: $R 2=.03, p<.01$.

Step 4: R2=.03, $p<.01$. Step 5: $R 2=.03, p<.01$.

Whereas, expressive suppression of emotion regulation appeared to be as the most significant predictor of psychopathic traits in male adolescents, $\beta=.40$, and parental acceptance-rejection (mother) in case of female adolescents, $\beta=.23$.

Table 4: Predictors of youth psychopathic traits in male adolescents $(n=281)$

\begin{tabular}{lccc}
\hline Model & B & SE & 8 \\
\hline Step 1 & & & \\
Expressive suppression & 1.45 & .20 & .40 \\
Step 2 & & & \\
Expressive suppression & 1.46 & .19 & .40 \\
\hline
\end{tabular}




\begin{tabular}{lccc}
\hline Psychological adjustment & .39 & .09 & .24 \\
Step 3 & & & \\
Expressive suppression & 1.43 & .19 & .39 \\
Psychological adjustment & .35 & .09 & .22 \\
Childhood trauma & .16 & .07 & .13 \\
Step 4 & & & \\
Expressive suppression & 1.18 & .21 & .32 \\
Psychological adjustment & .33 & .09 & .20 \\
Childhood trauma & .22 & .07 & .18 \\
Cognitive reappraisal & .47 & .16 & .17 \\
\hline
\end{tabular}

Step 1: $\mathrm{R} 2=.16, \mathrm{p}<.01$. Step 2: $\mathrm{R} 2=.21, \mathrm{p}<.01$.

Step 3: $R 2=.22, p<.01$. Step 4: $R 2=.24, p<.01$.

Table 5: Predictors of youth psychopathic traits in female adolescents $(n=319)$

\begin{tabular}{llll}
\hline Model & B & SE & 6 \\
\hline Step 1 & & & \\
Parental acceptance-rejection (mother) & .41 & .10 & .23 \\
Step 2 & & & .17 \\
Parental acceptance-rejection (mother) & .30 & .11 & .14 \\
Psychological adjustment & .19 & .08 &
\end{tabular}

Step 1: $\mathrm{R} 2=.05, \mathrm{p}<.01$. Step 2: $\mathrm{R} 2=.06, \mathrm{p}<.01$.

To determine the differences of psychopathic traits across adolescent's groups between-with in ANOVA was conducted for gender on psychopathic traits and its subsequent subscales.

Table 6: Between-within ANOVA for gender on youth psychopathic trait inventory and its subscale

\begin{tabular}{lrrrrrr}
\hline Sources of variance & $d f$ & $M S$ & $F$ & $P$ & $\eta^{2}$ & Observed Power \\
\hline Between Subjects & & & & & & \\
\hline Gender & 1 & 55.45 & 1.74 & .19 & .01 & .26 \\
Within Error & 598 & 31.83 & & & & \\
\hline Within Subjects & & & & & & 1.00 \\
\hline Subscales of YPI & 9 & 874.42 & 129.55 & .01 & .18 & 1.00 \\
YPI x Gender & 5 & 10.23 & 21.26 & .01 & .03 & \\
Within gender Error & 5382 & 6.75 & & & & \\
\hline
\end{tabular}

The result indicated a non-significant main effect of gender, $F(1,598)=1.74, p=n . s$ and a significant interaction between gender and all subscales of psychopathic tendencies, $F(5,5382)=21.26, p<.01$ and a highly significant main effect of the all subscales of psychopathic tendencies, $F(9,5382)=129.55, p<.01$. The data was broken down to determine gender differences across variables of YPI, PAQ, ERQ, CTQ and PARQ. Significant gender differences were not reported for YPI, $t(598)=1.34, p=n . s$. In case of PAQ, there had been a significant gender differences reported between two groups of adolescents, $t(598)=$ $-1.98, p<.05$. Likewise, for cognitive reappraisal of ERQ, there had been a highly significant gender differences reported among two groups of adolescents, $t(598)=-4.16 . p<.000$. However, differences were not significant for expressive suppression of ERQ, $t(598)=-1.16$. $p=n$.s. On the other hand, again there had been a highly significant gender differences reported among two groups on the measure of CTQ, $t(598)=10.96, p<.000$, for PARQ_F, the gender differences were highly significant $t(598)=3.95$, $p<.000$ and in case of PARQ_M, again the gender differences were highly significant, $t(598)=2.81, p<.001$.

Table 7: Gender wise comparison of YPI, PAQ, ERQ, CTQ, and PARQ

\begin{tabular}{lrrrr}
\hline Variables & Males & Females & \multirow{2}{*}{ t } & $p$ \\
\hline YPI & $M(S D)$ & $M(S D)$ & & 0.1 \\
PAQ & $109.28(18.57)$ & $107.33(17.18)$ & 6.17 & 0.05 \\
\hline
\end{tabular}




\begin{tabular}{lrrrr}
\hline Cognitive reappraisal (ERQ) & $25.74(6.84)$ & $27.99(6.44)$ & -4.16 & .000 \\
Expressive suppression (ERQ) & $18.05(5.11)$ & $18.55(5.51)$ & -1.16 & .247 \\
CTQ & $58.75(14.96)$ & $47.87(8.89)$ & 10.96 & .000 \\
PARQ_F & $38.49(8.99)$ & $35.37(10.19)$ & 3.95 & .000 \\
PARQ_M & $38.15(10.77)$ & $35.81(9.61)$ & 2.81 & 0.01 \\
\hline df $=598$ & & & &
\end{tabular}

Table 8: Analysis of the bio-data forms

\begin{tabular}{|c|c|c|c|}
\hline Variables & $\begin{array}{r}\text { Males } \\
281(46.8 \%) \\
f(\%)\end{array}$ & $\begin{array}{r}\text { Females } \\
319(53.2 \%) \\
f(\%)\end{array}$ & $\begin{array}{r}\text { Total } \\
600(100 \%) \\
f(\%)\end{array}$ \\
\hline \multicolumn{4}{|l|}{ Number of siblings } \\
\hline Single Child & $11(3.2)$ & $8(2.5)$ & $19(3.2)$ \\
\hline Two to Four & $149(53)$ & $193(60.6)$ & $342(56.9)$ \\
\hline Five or More & $121(43.1)$ & $118(36.9)$ & 239(39.9) \\
\hline \multicolumn{4}{|l|}{ Birth Order of the } \\
\hline \multicolumn{4}{|l|}{ Participants } \\
\hline Single Child & $11(3.2)$ & $8(2.5)$ & $19(3.2)$ \\
\hline Elder Child & $58(20.6)$ & $95(29.8)$ & $153(25.5)$ \\
\hline Middle Child & $142(50.5)$ & $145(45 \cdot 5)$ & $287(47.8)$ \\
\hline Younger Child & $72(25.6)$ & $71(22.3)$ & $143(23.8)$ \\
\hline \multicolumn{4}{|l|}{ Father's education } \\
\hline Uneducated & $17(6.0)$ & $17(5 \cdot 3)$ & $34(5.7)$ \\
\hline Matric & $138(49.1)$ & $104(32.6)$ & $242(40.3)$ \\
\hline Intermediate & $34(12.1)$ & $64(20.1)$ & $98(16.3)$ \\
\hline Bachelor & $44(15 \cdot 7)$ & $69(21.6)$ & $113(18.8)$ \\
\hline Masters & $38(13 \cdot 5)$ & $42(13.2)$ & $80(13 \cdot 3)$ \\
\hline Professional education & $10(3.6)$ & $23(7.2)$ & $33(15.5)$ \\
\hline \multicolumn{4}{|l|}{ Fathers' Profession } \\
\hline Govt. Servant & $89(31.7)$ & $79(24.8)$ & $168(28.0)$ \\
\hline Businessmen & $64(22.8)$ & $92(28.8)$ & $156(26.0)$ \\
\hline Private Service & $114(40.6)$ & $130(40.8)$ & $244(40.7)$ \\
\hline Dead & $14(5.0)$ & $18(5.6)$ & $32(5 \cdot 3)$ \\
\hline \multicolumn{4}{|l|}{ Mother's education } \\
\hline Uneducated & 49(17.4) & $28(8.8)$ & $77(12.8)$ \\
\hline Matric & $134(47.7)$ & $113(35.4)$ & $247(41.2)$ \\
\hline Intermediate & $22(7.8)$ & $81(25.4)$ & $103(17.2)$ \\
\hline Bachelor & $29(10.3)$ & $55(17.2)$ & $84(14.0)$ \\
\hline Masters & $39(13.9)$ & $37(11.6)$ & $76(12.7)$ \\
\hline Professional education & $8(2.8)$ & $5(1.6)$ & $13(2.2)$ \\
\hline \multicolumn{4}{|l|}{ Mother's profession } \\
\hline House wives & $249(88.6)$ & $289(90.6)$ & $538(89.7)$ \\
\hline Working & $32(11.4)$ & $11.30(9.4)$ & $62(10.3)$ \\
\hline
\end{tabular}

The analysis of bio-data forms was also conducted regarding the variables of birth order, number of siblings, father's and mother's education and father's and mother's profession of the participants. Mostly the participants were middle born, belonged to the families having two to four children, parents were matriculated, fathers belonged to multidimensional private services and mothers were housewives.

Insert Table 8 about here

\section{DISCUSSION}


The present study showed that as hypothesized, psychopathic tendencies are related with psychological adjustment, childhood trauma, perceived parental rejection and emotion regulation. It was explored that psychopathic tendencies were evident in both genders of adolescents with in the perspective of majorly a collectivistic society. Other important aspects regarding studying psychopathic tendencies with relation to other variables was to understand their cultural significance, intensity, factors contributing to them and lastly the risk management with regard to adolescents. The interaction and associations of different demographic variables with measures of research were also taken into account with respect to existing socio-cultural aspects of our society.

With respect to Pakistani culture, as the phenomena of psychopathic tendencies in relation to adolescents were not studied before so it was first explore that whether psychopathic tendencies were present in adolescents or not. The results indicted the presence of moderate to high level of psychopathic tendencies with in both genders. The analysis of the descriptive showed that majority of the adolescents with moderate range of psychopathic tendencies experienced low to moderate range of trauma and are moderate to highly emotion regulated with diverse range of experiences from psychological adjustment to psychological maladjustment.

Results also showed that psychopathic tendencies are evident in both genders but there are substantial differences in areas of interest and the level of experiences. Male adolescents had higher mean level in the dimensions of dishonest charm, grandiosity, lying, manipulation, remorselessness, unemotionally and irresponsibility, while females had greater mean on the dimensions of callousness, thrill-seeking and impulsiveness of psychopathic tendencies. Although that overall the differences between males and females was not significant but it is also important to consider that males showed higher mean scores on different dimensions of psychopathic tendencies as compared to female adolescents.

The above mentioned results provided the ground for investigating the relationships of psychopathic tendencies with childhood trauma, emotional regulation, parental acceptance/rejection (father \& mother) and psychological adjustment. Thus, the findings of the study showed that out of all the variables included in the study, psychological adjustment appeared to be as the most effecting predictor of psychopathic traits in adolescents, which indicated that higher level of psychological maladjustment of the adolescents in a given environment is likely to predict higher levels of psychopathic tendencies. Emotion regulation and childhood trauma also appeared to be as the other effecting variables on psychopathic tendencies. This finding is completely in line with the above mentioned results of analysis of descriptive. Higher the childhood trauma is likely to take to the development of higher psychopathic tendencies and in relation to it, lower the emotion regulation takes to higher level of psychopathic tendencies. As the findings of the study indicated, previous researches also supported the idea that that exposure to traumatic life experiences early in life make the child less sensitive to the pain of others and has the vulnerability to develop callousness, unresponsive to the needs of others and also lack empathy (Weiler \& Widom, 1996).

Furthermore, as the findings of the present research study showed, literature highlights that individuals with psychopathic tendencies may have very diverse patterns of responses to emotional stimuli, both of which could contribute to their problems in conscience development. Therefore, theories of moral development propose that patterns of emotional under-arousal and over-arousal may both get in the way with the normative conscience development (Frick \& Morris, 2004; Hoffman, 1982; Young, Fox, \& Zahn-Waxler, 1999). Available research has quoted this idea that individuals with psychopathic tendencies appear to exhibit differences in their emotional and cognitive processing (Newman, Patterson, Howland, \& Nichols, 1990; Newman \& Schmitt, 1998; Newman, Schmitt, \& Voss, 1997). It can be concluded that those adolescents who experienced traumatic events early in life are emotionally less regulated and so are highly vulnerable of the development of psychopathic tendencies later in their lives. The findings also indicated that emotion regulation appeared to be the most effecting variable in case of psychopathic tendencies in male adolescents. The other significant variables were childhood trauma and psychological adjustment. The results have the great cultural significance, as in Pakistan and generally in most of the collectivistic societies of the third world, violence becomes an intimidation against the social 
structure of society. As indicated by the reports of world health organization, there is a distressing increase in the incidence of mental illness due to a result of continuous wave of violence, political chaos and recurrent changes in the social fabric across many countries in the world (World Health Organization, 2001; 2005). A wide range of psychiatric disorders have been reported, as a result of such changes, like depression, substance and alcohol misuse, bipolar disorder, post-traumatic stress disorder, schizophrenia \& psychopathic tendencies (Gadit,2007).

Violence effecting all levels of individuals and especially adolescents who adopted it as way to work out their negativity. These emotionally less regulated adolescents are at high risk of developing psychopathic tendencies. The findings of the research also provide a link that emotionally deregulated individuals also have psychological maladjustment and exposure to traumatic childhood experiences. Cohen, Mannarino, and Deblinger (2006) have found that trauma during early childhood may have even greater consequences for developmental trajectories than traumas that occur in later adolescence. Some researchers have proposed that abuse is the result of reduced capacity of an individual to respond with empathy and at edge of developing psychopathy later in life.

The results further indicated that perceived rejection by mother appeared to be as the most effecting variable on psychopathic tendencies in female adolescents. The other significantly effecting variable was psychological adjustment. The findings are again supported by the socio-cultural perspective, where females are perceived to be highly bonded with their mothers and has less exposure to the wide range of experiences. In such perspective, perceived rejection by the mothers may have tremendous developmental consequences for the female adolescents and make them vulnerable for developing psychopathic tendencies. The findings of the study are supported by the previous research, Vural, Hafızoğlu, Türkmen, Eren, and Büyükuysal (2012) suggested that there was a significant correlation existed among child and mother reports of perceived acceptance. The perceived rejection by the mothers also effect the psychological adjustment of an individual. Thus the findings also indicated that perceived rejected female adolescents are highly susceptible for developing psychopathic tendencies and also psychologically maladjusted to their environment.

Furthermore, the main effect of gender (males \& females) was not indicated as significant for psychopathic tendencies but the analysis of the subscales showed that all the subscales were perceived in a different way by male and female adolescents. The findings of this study are contrary to the previous researches which showed that men have higher level of psychopathic tendencies then women (Cale \& Lilienfeld, 2002a; Verona \& Vitale, 2006; Vitale \& Newman, 2001). It is important to mention that most of the researches are conducted with adults and not with adolescents, so it may possible that in adolescence, the same levels of tendencies are present in both genders but later in life men get more conducive environment as compare to women to develop their tendencies. Women in collectivistic societies are usually suppressed in expression of their emotions so it can be concluded through the findings of this research that women are at risk of developing psychopathic tendencies equally like men later in their life depending on the cultural norms and values, as the psychopathy is highly effected by the norms and values prevailing in a society (Draguns, 1973).

The qualitative analysis of the bio data form revealed that most of the male adolescents belonged to extended families having five or more siblings, while the female adolescents belonged to those families having two to four siblings. Birth order of the both male and female participants was middle born. While fathers were also not significantly educated in case of both genders. Fathers were usually matric and mostly belonged to private services (i.e., day paid laborers). Mothers of both genders were also having matric as their education qualification in both genders and the vast majority was housewives.

The in-depth analysis of the bio data form provides a clear picture that how much vulnerable environment is for adolescents who are at risk of developing psychopathic tendencies. As they live in extended families, this can be significantly translated in terms of care given by the parents to their children. The families with many children leave the parents with divided attention and care. Some children may need special care at times which the parents are unable to provide. This is also related with the presumption, 
mentioned earlier, that this lack of care may be the root cause of a confused relationship between parents and child which leads to a lack of understanding and intimacy on both sides. In the socioeconomic condition of our country, a larger family means that the factor of education may be compromised. As education is directly related with the understanding of different psycho-emotional problems of adolescents. This may be a contributing factor. As the parents of large portion of the sample was not significantly educated so that it can be placed a question mark on its functional contribution in an adolescent life when he/she is experiencing the most crucial part of his/her life.

Overall it may be concluded that psychopathic traits, psychological adjustment and emotional regulation, parental acceptance/rejection (father \& mother forms) and childhood trauma are all inter-correlate. There were significant gender differences on different measures of research.

\section{LIMITATIONS AND SUGGESTIONS}

Some limitations did not permit the investigation of many aspects that could be of great value.

- The sample was restricted to those schools of Lahore, Gujranwala and Sialkot which granted permission for conducting research with students. The data may be collected from the whole country for the sake of generalizability of the findings to include different ethnicities and races as well.

- Qualitative analysis should be incorporated because of the subjectivity of the many factors involved.

\section{CONCLUSION}

Adolescence is the most vulnerable period of human life where in the presence of conducive circumstances many such underlying factors like psychopathic tendencies can totally distort the personality of an adolescent. It has been showed through the present research that the presence of childhood trauma, with other contributing factors like psychological maladjustment and emotional dysregulation along with perceived rejection by parents are all interrelated and can contribute in establishing the psychopathic tendencies in the vulnerable individuals. The findings of this research study are in line with the findings of the previous studies dealing with the same variables, so validating the findings of other researches with in Pakistani cultural perspective. However, contrary to previous research, within our socio-cultural perspective, both genders are at equal risk of exhibiting developing psychopathic tendencies. It is also quite alarming that out of the data of 600 adolescents, majority were having moderate psychopathic tendencies which can lead to development of higher psychopathic tendencies. This provide an important reason to carry out further follow-up studies taking broader perspective by increasing the data, geographical areas, different ethnicities and incorporating other important demographic variables with in the perspective of Pakistan. It is also important to take such studies world widely with the sample of normal population, as most of such studies in all the cultures are conducted with offenders and criminals, so to develop a mechanism for dealing and helping individuals who are at risk. It's important to educate the teachers, parents and overall society that to take it as their responsibility to rescue such individuals whose mental health is at stake and also to communicate the findings of present study at different forums to increase the awareness level regarding this underlying serious matter.

\section{REFERENCES}

Ajdukovic, M. (1990). Differences in parent's rearing style between female and male pre-delinquent and delinquent youth. Psychologische Beitrage, 32, 7-15.

Al-Falaij, A. (1991). Family conditions, ego development and socio-moral development in juvenile delinquency: A study of Bahraini Adolescents. (Unpublished doctoral dissertation). University of Pittsburgh, PA. 
Andershed, H. (2010). Stability and change of psychopathic traits: What do we know? In D. Lynam \& R. Salekin (Eds.), Handbook of child and adolescent psychopathy. New York, NY: Guilford.

Andershed, H., Kerr, M., Stattin, H., \& Levander, S. (2002). Psychopathic traits in non-referred youths: Initial test of a new assessment tool. In E. Blaauw, J.M. Philippa, K. C. M. P. Ferenschild, \& B. van Lodensteijn (Eds.), Psychopaths: Current international perspectives (pp. 131-158). The Hague: Elsevier.

Baker, C.N., \& Hoerger, M. (2012). Parental child-rearing strategies influence self-regulation, socioemotional adjustment, and psychopathology in early adulthood: Evidence from a retrospective cohort study. Personality and individual differences, 52, 800-805.

Belsky, J., \& Pensky, E. (1988). Developmental history, personality, and family relationships: Toward an emergent family system. In R. A. Hinde, \& J. Stevenson-Hinde (Eds.), Relationships within families Oxford (pp. 193-217). Clarendon Press.

Bernstein, D.P., Fink, L., Handelsman, L., Foote, J., \& Lovejoy, M. (1994). Initial reliability and validity of a new retrospective measure of child abuse and neglect. Am J Psychiatry, 151, 1132-1136.

Bernstein, D.P., Stein, J.A., Newcomb, M.D., Walker, E., \& Pogge, D. (2003). Development and validation of a brief screening version of the Childhood Trauma Questionnaire. Child Abuse Negl, 27, 169-190.

Butt, M.M., Khan, S., Malik, F., \& Sharif, M.J. (2013). Translation of Childhood Trauma Questionnaire. Copyright 1996, David P. Bernstein, \& Laura Fink.

Butt, M.M., Malik, F., \& Kaleem, Z. (2012). Translation of emotion regulation questionnaire. Copy rights reserved by gross \& john.

Butt, M.M., Saeed, M., Walayat, S., \& Malik, F. (2013). Translation of youth psychopathic traits inventory. Copyrights reserved by Henrik Andershed, Orebro University, Sweden.

Cale, E. M., \& Lilienfeld, S. O. (2002a). Histrionic personality disorder and antisocial personality disorder: Sex differentiated manifestations of psychopathy. Journal of Personality Disorders, 16, 52-72. doi:10.1521/pedi.16.1.52.22557

Caspi, A., Taylor, A., Moffitt, T. E., \& Plomin, R. (2000). Neighborhood deprivation affects children's mental health: Environmental risks identified in a genetic design. Psychological Science, 11, 338-342.

Chen, X., Rubin, K.H., \& Li, D. (1997). Maternal acceptance and social and school adjustment: A four-year longitudinal study. Merrill- Palmer Quarterly, 43, 663-681.

Cleckley, H. (1941). The mask of sanity: An attempt to reinterpret the so-called psychopathic personality. Oxford, UK: Mosby (Original work published 1988).

Cohen, J. A., Mannarino, A. P., \& Deblinger, E. (2006). Treating trauma and traumatic grief in children and adolescents. New York, NY: Guilford Press.

Coupland, R., \& Moretti, M. M. (2010). Gender Differences in the relationships between maternal and paternal physical abuse in the development of psychopathy. Paper presented in a Symposium at the Biennial Meeting of the American Psychology-Law Society, Vancouver, BC.

Crook, T., Raskin, A., \& Eliot, J. (1981). Parent-child relationships and adult depression. Child Development, 52, 950-957.

David, D.B., \& Malik, M.H. (2000). Stress and psychiatric disorder in Urban Rawalpindi, community survey. The British Journal of Psychiatry, 177, 557-62.

Donahue, J., Mcclure, K. S., \& Moon, S.M. (2014). The relationships between emotion regulation difficulties and psychopathic personality characteristics. Personality disorders: theory, Research, and treatment, 5(2),186-194. doi: 10.1037/peroo000025

Draguns, I. G. (1973). Comparison of psychopathology across cultures: Issues, findings, directions. Journal of Cross-Cultural Psychology, 4, 9-47.

Dumka, L. E., Roosa, M. W., \& Jackson, K. M. (1997). Risk, conflict, mothers' parenting, and children's adjustment in low-income, Mexican immigrant, and Mexican American families. Journal of Marriage and the Family, 59, 309-323.

Farrington, D. P., \& Hawkins, J. D. (1991). Predicting participation, early onset and later persistence in officially recorded offending. Criminal Behavior and Mental Health, 1, 1-33.

Frick, R. W. (1995). A problem with confidence intervals. American Psychologist, 50, 1102-1103.

Frick, P. J., \& Morris, A. S. (2004). Temperament and developmental pathways to conduct problems. Journal of Clinical Child and Adolescent Psychology, 33, 54-68.

Gadit, A.A.M. (2005). Disaster, mental health and rescuing medical professionals. Journal of Ayub Medical College Abbottabad, 17(4), 1-2. 
Gadit, A.A.M. (2007). Mental health model: comparison between a developed and a developing country. Journal of Medicine, 1(1).

Gadit, A.A.M., \& Vahidy, A. (1999). Mental health morbidity pattern in Pakistan. Journal of College of Physicians and Surgeons Pakistan, 9, 362-65.

Galea, S., Ahern, J., Resnick. H., Kilpatrick. D., Bucuvalas, M., \& Gold, J. (2002). Psychological sequelae of the September 11 terrorist attacks in New York City. New England Journal of Medicine, 346, 982-7.

Galea, S., Vlahov, D., Resnick, H., Ahern, J., Susser, E., \& Gold, J. (2003). Trends of probable post-traumatic stress disorder in New York City after the September 11 terrorist attacks. American Journal of Epidemiology, 158(6), 514-24.

Greenberger, E., \& Chen, C. (1996). Perceived family relationship and depressed mood in early and late adolescence: A comparison of European and Asian Americans. Developmental Psychology, 32, 707716.

Hare, R. D. (2003). The Hare Psychopathy Checklist-Revised ( $2^{\text {nd }}$ ed.). Toronto, Ontario, Canada: MultiHealth Systems.

Hare, R.D., McPherson, L. M., \& Forth, A. E. (1988). Male psychopaths and their criminal careers. Journal of Consulting and Clinical Psychology, 56, 710-714.

Hart, S. D., Kropp, P.R., \& Hare, R. D. (1988). Performance of male psychopaths following conditional release from prison. Journal of Consulting and Clinical Psychology, 5, 227-232.

Hart, S.D., Watt, K., \& Vincent, G. (2002). Commentary on Seagrave and Grisso: Impressions of the state of the art. Law and Human Behavior, 26, 241-246. doi:10.1023/A:1014648227688

Hoffman, M. L. (1982). Development of prosocial motivation: Empathy and guilt. In N. Eisenberg (Ed.), The development of prosocial behavior (pp. 281-313). San Diego, CA: Academic Press.

Jacobson, S., Fasman, J., \& DiMascio, A. (1975). Deprivation in the childhood of depressed women. The Journal of Nervous and Mental Disease, 160, 5-14.

Khalily, T.M., Fooley, S., Hussain, I., \& Bano, M. (2011). Violence, psychological trauma and possible acute post-traumatic interventions in Pakistani society. Australasian Journal of Disaster and Trauma Studies, $1,1-9$.

Lynam, D. R. (1996). The early identification of chronic offenders: Who is the fledgling psychopath? Psychological Bulletin, 120, 209-234. doi: 10.1037//0033-2909.120.2.209

Malik, F. (2011). Translation of Parental acceptance-rejection questionnaire (child PARQ). Copyright @ 1976, 1999, 2004 by Rohner Research Publications All rights reserved (Revised June, 2004)

Marzuk, P. (1996). Violence, crime, and mental illness. How strong a link? Archives of General Psychiatry, $53,481-6$.

Maughan, B., Pickles, A., \& Quinton, D. (1995). Parental hostility, childhood behavior, and adult social functioning. In J. McCord (Ed.), Coercion and punishment in long-term perspectives. Cambridge: Cambridge University Press.

Moffitt, T. E. (1993). Adolescence-limited and life-course persistent antisocial behavior: A developmental taxonomy. Psychological Review, 100, 674-701.

Naz, F., \& Kausar, R. (2011). Translation of Personality assessment questionnaire (child PAQ). Copyright @ 1976, 1999, 2004 by Rohner Research Publications All rights reserved (Revised June, 2004).

Newman, J. P., Patterson, C. M., Howland, E. W., \& Nichols, S. L. (1990). Passive avoidance in psychopaths: The effects of reward. Personality and Individual Differences, 11, 1101-1114.

Newman, J. P., \& Schmitt, W. A. (1998). Passive avoidance in psychopathic offenders: A replication and extension. Journal of Abnormal Psychology, 107, 527-532.

Newman, J. P., Schmitt, W., \& Voss, W. (1997). The impact of motivational neutral cues on psychopaths: Assessing the generality of the response modulation hypothesis. Journal of Abnormal Psychology, $106,563-575$.

Pedersen, W. (1994). Parental relations, mental health, and delinquency in adolescents. Adolescence, 29, 975-990.

Rohner, R. P. (1975). They Love Me, They Love Me Not: a worldwide study of the effects of parental acceptance and rejection. In Ronald P. Rohner (Ed) The Warmth Dimension, foundation of parental acceptance-rejection theory. Storrs, CT: Rohner Research Publications.

Rohner, R. P. (1990). Handbook for the study of parental acceptance and rejection (3rd ed.). Storrs, CT: Rohner Research Publications. 
Rowling, L. (2006). Adolescents and emerging adulthood (12-17 years and 18-24 years). In A. Cattan \& S. Tilford (Eds.), Mental health promotion: A lifespan approach (pp. 100-136). New York: McGraw-Hill,

Salama, M. (1984). Child rearing methods in relation to the psychological problems in middle childhood. (Unpublished doctoral thesis). Ain Shams University, Cairo, Egypt.

Salekin, R., Rogers, R., \& Sewell, K. (1996). A review and meta-analysis of the Psychopathy Checklist and Psychopathy Checklist-Revised: Predictive validity of dangerousness. Clinical Psychology: Science and Practice, 3, 203-215. doi:10.1111/j.1468-2850.1996.tboo071.x

Saluja, G., lachan, R., Scheidt, P. C., Overpech, M. D., Sun, W., \& Gidd, J. N. (2004). Prevalence of and risk factors for depressive symptoms among young adolescents. Archives of General Psychiatry, 158, 760765.

Saxena, V. (1992). Perceived maternal rejection as related to negative attention-seeking classroom behavior among primary school children. Journal of Personality and Clinical Studies, 8, 129-135.

Shahid, S, F., Mullick, M.S.I., Nahar, J.S., Naher, J., Khan, S., Morshed, N, M., Shah, M, A., \& Qusar, M.M.A.S. (2009). Relationship between parental rejection and personality. Bangabandhu sheikh mujib medical university journal, 2(2), 61-65.

Shipman, K. L., Schneider, R., Fitzgerald, M.M., Sims, C., Swisher, L., \& Edwards, E. (2007). Maternal emotion socialization in maltreating and non-maltreating families: Implications for children's emotion regulation. Social Development, 16(2), 268-285.

Slemming, K., Sorensen, M. J., Thomsen, P. H., Obel. C., Henriksen, T. B., \& Linnet, K. M. (2010). The association between preschool behavioural problems and internalizing difficulties at age 10-12 years. European Child and Adolescents Psychiatry, 19, 787-795.

Smith, L. (2011). An exploration of the relationship between poor parent-child attachment and callousunemotional traits in a sample of high-risk young offenders. (Unpublished doctoral thesis). Department of Clinical, Educational, and Health Psychology, University College London.

Verona, E., \& Vitale, J. (2006). Psychopathy in women: Assessment, manifestations, and etiology. In C. J. Patrick (Ed.), Handbook of psychopathy (pp. 415-436). New York, NY: Guilford Press.

Vitale, J. E., \& Newman, J. P. (2001). Using the Psychopathy Checklist- Revised with female samples: Reliability, validity, and implications for clinical utility. Clinical Psychology: Science and Practice, 8, 117132. doi:10.1093/clipsy.8.1.117

Vural, P., Hafizoglu, S., Turkmen, N., Eren, B., \& Buyukuysal, C. (2012). Perceived parental acceptancerejection and psychopathology in a group of sexually abused children/adolescents. Medicinski glansik, 9(2), 363-369.

Whitbeck, L.B., Conger, R.D., \& Kao, M. (1993). The influence of parental support, depressed affect, and peers on the sexual behaviors of adolescent girls. Journal of Family Issues, 14, 261-278.

Whitbeck, L. B., Hoyt, D. R., Miller, M., \& Kao, M. (1992). Parental support, depressed affect, and sexual experience among adolescents. Youth and Society, 24, 166-177.

Weiler, B., \& Widom, C. S. (1996). Psychopathy and violent behavior in abused and neglected young adults. Criminal Behavior and Mental Health, 6, 253-271.

World Health Organization (2001). World health report: global burden of disease. Geneva, Switzerland: WHO; 2001.

World Health Organization (2005). Mental Health Atlas 2005 (revised edition). Geneva, Switzerland: WHO; 2005.

Young, S. K., Fox, N. A., \& Zahn-Waxler, C. (1999). The relations between temperament and empathy in 2-year-olds. Developmental Psychology, 35, 1189-1179. 